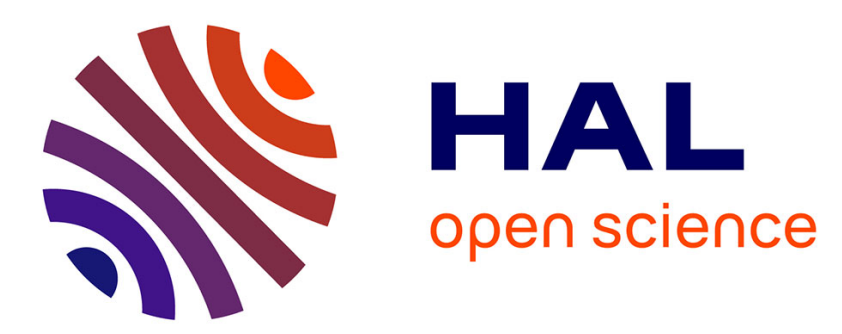

\title{
Asymptotic probabilities of languages with generalized quantifiers
}

\author{
Guy Fayolle, Stéphane Grumbach, Christophe Tollu
}

\section{To cite this version:}

Guy Fayolle, Stéphane Grumbach, Christophe Tollu. Asymptotic probabilities of languages with generalized quantifiers. Eighth Annual IEEE Symposium on Logic in Computer Science, Jun 1993, Montréal / Canada, pp.199-207, 10.1109/LICS.1993.287587 . inria-00077192

\section{HAL Id: inria-00077192 https://hal.inria.fr/inria-00077192}

Submitted on 29 May 2006

HAL is a multi-disciplinary open access archive for the deposit and dissemination of scientific research documents, whether they are published or not. The documents may come from teaching and research institutions in France or abroad, or from public or private research centers.
L'archive ouverte pluridisciplinaire HAL, est destinée au dépôt et à la diffusion de documents scientifiques de niveau recherche, publiés ou non, émanant des établissements d'enseignement et de recherche français ou étrangers, des laboratoires publics ou privés. 


\section{Asymptotic probabilities of languages with generalized quantifiers}

GuY FAYOLLE

Sićphanc GRUMBACH

Christophe TOLLU

$$
\begin{aligned}
& N^{\circ} 1916 \\
& \text { Mai } 1993
\end{aligned}
$$

PROGRAMME 1

Archilcclures parallèles,

balses de données,

rescaux el systèmes distribués

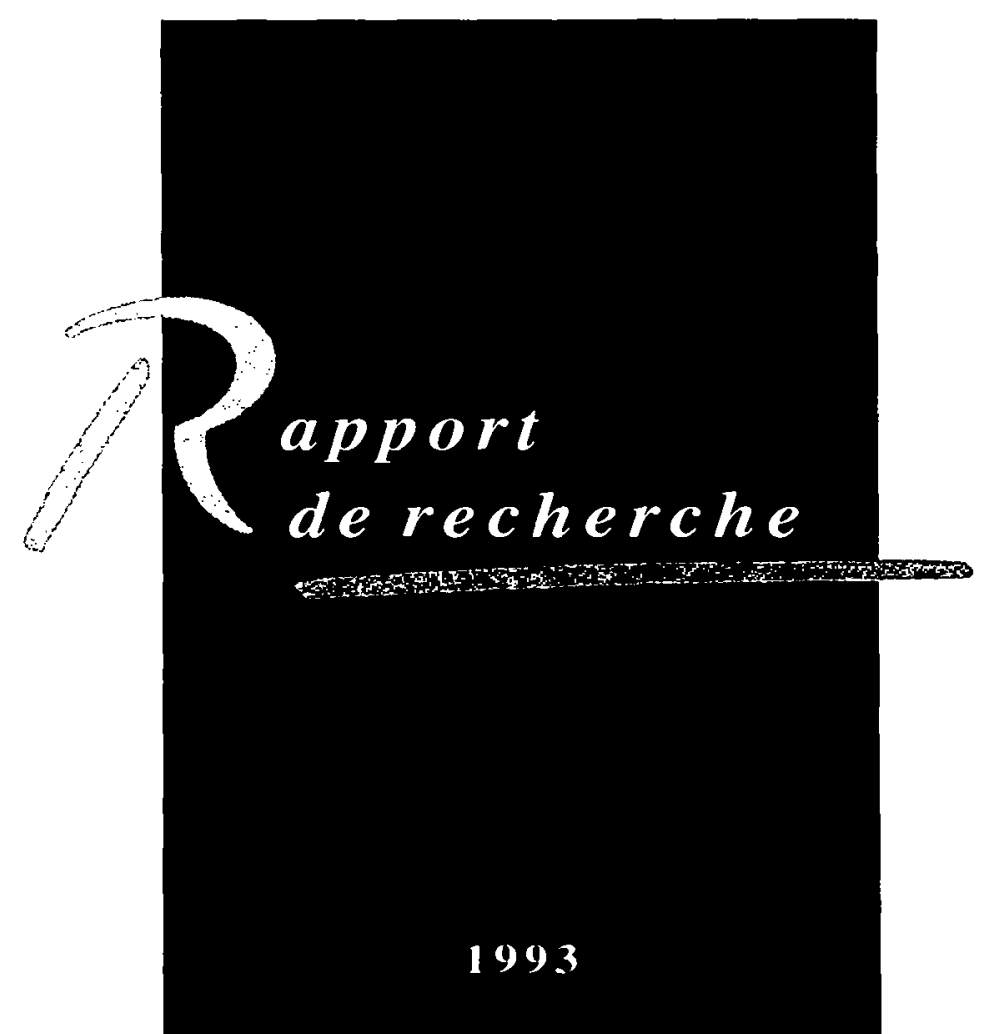




\title{
Probabilités Astmptoticunes de Langages arec des Ouantificateurs Crénćlalisés*
}

\author{
Guy Farolle Stephane Grumbach ('hristophe Tollu \\ I.A.R.I.A. \\ Rocquencourt BP 105 \\ TEl1:3 Le Chesnay \\ France \\ guy.fayolle iinria.fr \\ stephame.grmmbach gimria.fr \\ L.I.P.N. \\ IRRA 1507. Université Paris-Niorel \\ 9:34:30 Villotaneuse \\ France \\ ct(îlipn.univ-paris13.fr
}

\section{Résumé}

Nous étudions liffrt sur le comportement asymptotique des énoncés. de lajout de certaines familles de quantifirateurs grúralisés à la logique du premier ordre. Tous nos rósullats sont ́́tablis pour des langages sans variable libre à l'intérieur des quantificateurs grinćralinés. Pour une classe $\hat{N}$ de structures finics formée par isomorplisme. Ie quantificateur $Q_{\mathcal{K}}$ est dit fortement monotone si le problème d'apprivtenamer à la classe est preserlé pour une forme souple d'extensions. Notre promire thrórime (loi $0 / 1$ pour la logique du premier ordre aver un ensemble quelrongur de quanlifirateurs fortrment monotones) généralise un critère connu pour monterer que presque. aucun graphe ne satisfait une propriété donnée. Nous montrons aussisi une loi o/ 1 pour la logique du premier ordre avec des quantificateurs de Ilärtig (quantifirateurs d'óquicardinalité) et une loi limite pour un fragment de lo logique du premier ordre alece des quantificuteurs de Rescher (exprimant des inégalités entre les cordinalités). Les preures de ces deux derniers résultats font apurl i l'ínumération combinatoire standard et à des tcchniques plus sophlistigures dianalyse complere. Wous montrons aussisi que la loi $0 / 1$ n'est pas rérifiér prour une exterision de la logique du premier ordre aver des quantificateurs de Ilärtig si les restrirtions syntaxiques mentiomnées ci-dessus sont

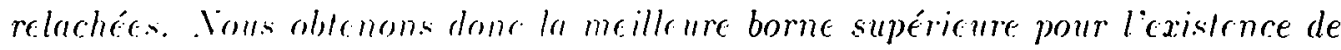
loi $0 / 1$ arere des quantifieratems de Itärtig.

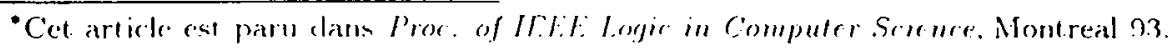




\section{Asymptotic Probabilities of Languages with Generalized Quantifiers}

\author{
Guy Fayolle Stéphane Cirumbach
}

\author{
I.X.R.I.I. \\ Rocquencourt BP 10; \\ 78153 Le Chesmay. \\ France \\ guy.fayolle.stephare.grmmach cimria.fr
}

('hristophe lollu

\author{
L.I.P.N. \\ URA 1507. Iniversité Paris- Nord \\ 9:3.130 Villetancuse \\ France \\ (ct)lipn.univ-paris $13 . \mathrm{fr}$
}

May 3. 199:3

\begin{abstract}
We study the impact of adding certam fammlies of generalized quantifiers to first-order logis: (FO) on the asymptotic beharior of sentences. Hll our if.ult, are statcd and proved for languages disallowm fire rariables in the scope of generalised quantifiers. For a class $\mathcal{K}$ of finite structures rloserl under esomorphesm. the quantifier $Q_{\mathcal{K}}$ is sard to be strongly monotonic. $\mathrm{sin}$. if membership in the cloms is preserved under a

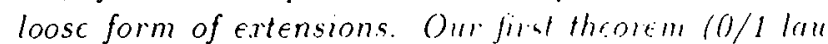
for FO with any set of $\mathrm{ill1}$ (qurontifiter) sulusumes a pretious critcrion for proting that alment no graphs satisfy a given property [BHrio]. The allow estableat a

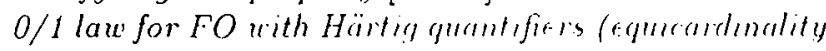
quantifiers) and a limit law for a fingment of $F O$ with

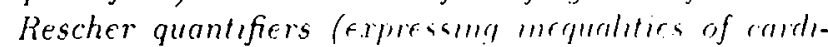
nalities). The proofs of the at last tero malls combine standard combinatoral enumfratuons will more sophisticated terhnigues from compler amalinss. It: also prove that the $0 / 1$ lare fail. for the erfenserion of

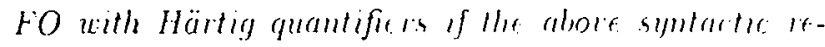
striction is relared. We them form grt the best upper bound for the eristence of a $\mathrm{O} / \mathrm{I}$ law for $\mathrm{FO}$ wth Härteg quantifiers.
\end{abstract}

\section{Introduction}

The idea of extending first-order loggie by means of generalized guantifiers clates bark to the work of

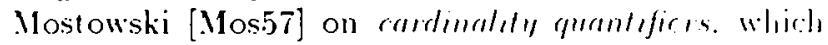
was an attempt to remely the fart that key notions of modern mathematics. such as the notion of a fi- nite set or the notion of an uncountable set, were not first-order definalyle over the class of all (either finite or infinite) structures. In Mostowski's stride. miscellaneons quantifiers inspired by probabilistic or topological concepts. came to light. A decade later, Lindström [Lin66] gave a very general definition of a quantifier. allowing practically any class $\mathcal{K}$ of structures to be used for defining a new quantifier $Q_{\mathcal{K}}$ that captures membership in that class. Since then. the study of languages with added quantifiers has been an important line of research of abstract modd theory [BF8.5].

Meanwhile. fimt model theory had emerged as an important research area [Fag90]. The steadily growing interest of logicians in finite structures was a consequence of the strengthened connections between logic and computer scicuce. Researchers rapidly realized that first-order logic (FO) was not timed properly for this new challenge. In particular. FO lacks any form of recursion mechanism that reveals necessary to define nsial properties of finite strurtures. For the last wo drades. a considrable amount of work has been achieved. in the context of finite model theory, on logirs wlowe expressive power surpanes fO's: Gurerich and shelah [Cis\&6]: among ollors, investigated and compared warous fixed-point extemsions of first-


underesok a carrfol cxamnination of infintary langunages. Vost of the work on extenderl logics over finite st rurburs was related to important problents of desritive complexity. Vory reently. gencralized quantifire haw berell sturdied in the realun of finite struetures

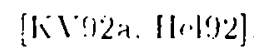

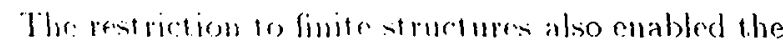
drsign and development of spreilie methods, among 
which 0/1 laws appear as ermeral. 'Tluis line of re-

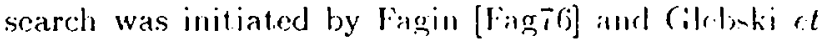
alii [GKIT69] who independrntly proved llor frellewing startling result: given any $\mathrm{FO}$ sontener $\hat{r}$. if all

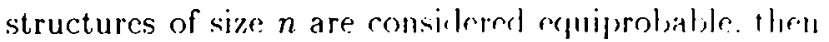
the limit, as $n \rightarrow \infty$, of the probability that $=$ is satisfied by a random strurture of size $n$. always exist.s and is equal to cither 0 or 1. Ianguages mujoying such a property are said to have a $0 / 1$ law. By now, the 0/1 law has becn shown to hold for mumerous extensions of first-order logir withont functions or constants: fixed-point logirs [HCiK85, KV87]. the infinitary logic with a finite number of variables $L u$ [KV90b] and some prenex classes of existential serondorder logic [K $\mathrm{K} 90 \mathrm{a}]$.

Our aim in the present paper is to study the asymptotic behavior of sentences of $F O(Q)$. where $Q$ is a set of generalized quantifiers. Wr first focus on strongly monotonic quantifiers. A quantifier $Q$. binding one formula, is strongly monotonic if it is defined by a clacs $\hat{\Lambda}$ of structures $\langle A, X\rangle$, with $\mathcal{X} \subseteq A^{k}$ for some integer $k$, that is preserved under a loose form of extension: $\langle A, X\rangle \in \mathcal{K}, A \subseteq B$ and.$Y \subseteq Y$ m ntail $\langle B\rangle.\rangle \in \mathcal{K}$. Strong monotonicity is a neat framework for capturing numerous classes of graplss, among which planarity. ncolorability, and so on. We give a simple proof that the $0 / 1$ law holds for the logirs $\mathrm{FO}^{*}(\mathrm{Q})$ extending (without free variables in the scope of generalized quantifiers) FO by any family of strongly monotonic quantifiers. Hence, we yield a generalization of a criterion of Blass and Harary [BH79] for proving that almost no graplis satisfy some propertios

We then turn to extensions of FO with rounting. To our knowledge, the only result on the asymptotic probabilities of extensions of first-order logic with counting can be found in [Kny90]. where it is proved that, for a rational $r$ such that $0 \leq r \leq 1$. if the asymptotic probability of a formula $\varphi(x)$ is rlifferent from $r$. sentences of the form: "there is at Irast a fraction $r$ of the elements of the domain satisfying $r(x)^{*}$ have a $0 / 1$ law. The restriction on $r$ is crucial. Indeed. he sentence expressing that "there is at frast one half of the elements of the domain satisfying $P(x)$ ". Where $P$ is some mary. predicate, has asymptotic prohahility $\frac{1}{2}$. Our results differ from Kinyazev's in two resperts: (i) we are contcerned with families of quam ifiers expressing equalities or incqualitics of first-oreler definable relations of arbitrary aritics. and (ii) nur procks roly upon raclically defferent methods. namely the I.aplace method and the saddle-point methol for computing intergals. It

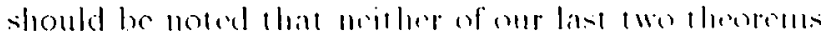

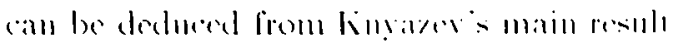

('ounting is an essential primitive in database query lingliages. Logical languages generally lack the ability 16 express rounting. though it is very easy to count on any computational device [AV91]. 0/1 laws have berll userl in this context to get upper bounds on the expressive power of query languages. These results give a better understanding of the expressive power gainer with commting primitives such as Härtig's and Rescher's quantifiers.

The paper is organized as follows. In the next seclion. we review the main definitions. Section 3 is deintel to strongly monotonic quantificrs. In Sections 4 and 5. two theorms are proved, which establish a $0 / 1$ law (respectively a limit-law) for extensions of FO by means of the Harrig quantificrs (respectively the Rescher (quantifiers).

\section{Asymptotic Probabilities and Gen- eralized Quantifiers}

In this section. we introduce the basic concepts and tools that will be used throughout the paper.

Let $\left\langle R_{1} \ldots R_{k}\right\rangle$ be a purely relational schema and $\tau=\left\langle r_{1} \ldots r_{k}\right\rangle$ be its sumilarily t!ne. i.e. the arity of $R$, is $r_{1}$. Strur<u[r] and $S t r u c_{n}[\tau]$ rospectively denote the rlass of finite $r$-structures and the class of $\tau$-structures of domain $n=\{0 \ldots n-1\}$. If $\mathcal{L}[\tau]$ is a logic for struc<w $[\tau]$ and $\dot{r}$ is a sentence of $\mathcal{L}[\tau] \cdot \mu_{n}(r)$ denotes the proportion of structures of Struc $[T]$ which satisfy $\hat{r}$ :

$$
\mu_{n}(\hat{r})=\frac{\left|\left\{\mathcal{A} \in S t r u c_{n}[\tau] \mid \mathcal{A} \models \tau\right\}\right|}{\left|S t u c_{n}[\tau]\right|} .
$$

The asumptoter mobriblety $\left.\mu_{(}\right)$of $r$ is the limit. if it. exists. of $\mu n(r)$. as $n \rightarrow x$. A property is almost surlu iass) trut (resp. almost surrly false) if its astmptotic probability is 1 (rosp. 0). If the asympuric probability is defined for every sentence of $\mathcal{L}[\tau]$, $L[r]$ is said to have a limil loue. If. in addition. the as rmomptoce probability is either 0 or $1 . \mathcal{L}[\tau]$ is said to have a (lalelled) O/l lau.

In [Fagio]. Fagin proved that there is a $r$-structure over the set is of all illtegers. called the random rountable - -sfructure, which plays a key role in the study of the asymptic probabilities of $\mathrm{FO}[\tau]$ sentences. In the sepuel. $\Omega[r]$ will denote the random countable r-stmeture. The following well-known property will turn out 10 be esintuial to the proofs of Theorems 4.1 , 5.1. 
Proposition 2.1 [Fagfoi] for arengl $\geq 1$, Ihr mmm-

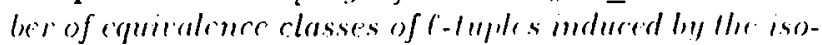
morphisms of $\Omega[\tau]$ is fimle and rqual to $N_{1}(\tau)$. whrh depends only upon $\ell$ and llw similarily type $\tau$. Furthermore,

- each equitalence class (" is rhametemerd on $\Omega[T]$ by a $F O[T]$ sentence willhout quantifier (a romplete open description). $\Phi_{r} \cdot\left(m_{1}, \ldots, x_{r}\right)$, t.e. for each $r$-tuiple $\left\langle a_{1}, \ldots a_{r}\right\rangle$ of integress.

$$
\left\langle a_{1}, \ldots, a_{\ell}\right\rangle \in C^{\prime} \text { iff } \Omega[r] \models \Phi_{c}\left(a_{1} \ldots, a_{f}\right) \text {; }
$$

- each equiralcnce class (' is equiprobable, i.e. for a random P-tuple $\bar{a}$.

$$
\operatorname{Prob}(\bar{a} \in C)=\frac{1}{N_{c}(\tau)}
$$

- each $F O[\tau]$ formula wilh f fire variables $\varphi(\bar{x})$ is asymptotically equivalent to a union of equicalence rlasses of C-tuples. i.f. there exist classes $C_{1}, \ldots, C_{r}$, such that:

$$
\lim _{n \rightarrow \infty} \forall \bar{x}\left(\varphi(\bar{x}) \Leftrightarrow \bigvee_{1 \leq i \leq p} \Phi_{C_{1}}(\bar{x})\right)=1 .
$$

Let $\mathcal{K}$. be a class of $\tau$-structures closed under isomorphism. We associate $\mathcal{N}$ with a generalized quantifier $Q_{\mathcal{K}}$, of type $\tau$. $\mathrm{FO}\left(Q_{\mathcal{N}}\right)$ is the logic extending $\mathrm{FO}$ by the following formation rule.

- If $\varphi_{1}, \ldots, \varphi_{k}$ are formulas and, for each $i \in$ $\{1, \ldots, k\}, \bar{x}_{i}$ is a tuple of variables of arity $r_{i}$. then $Q_{K} \bar{x}_{1}, \ldots, \bar{x}_{k}\left(\hat{y}_{1}\left(\bar{x}_{1}\right) \ldots, \varphi_{k}\left(\bar{x}_{k}\right)\right)$ is a formula. Note that $Q_{\kappa}$ binds all occurrences of variables from $\bar{x}_{i}$ in $\varphi_{i}$.

The semantics of $Q_{\mathcal{K}}$ is defined by:

- $\mathcal{A} \vDash \quad Q_{\boldsymbol{N}} \bar{x}_{1} \ldots, \bar{x}_{k}\left(\bar{r}_{1}\left(\bar{x}_{1}\right), \ldots, \varphi_{k}\left(\bar{x}_{k}\right)\right)$ iff $\left\langle A, \varphi_{1}^{\mathcal{A}}, \ldots, \varphi_{k}^{\mathcal{A}}\right\rangle \in \mathcal{N}$.

where $A$ is the domain of $A$

and $\varphi_{i}^{\mathcal{A}}=\left\{\bar{a} \in A^{r_{i}} \mid \mathcal{A} \models \xi_{i}(\bar{a})\right\}$.

Obviously, for any set $Q$ of generalized quantifiers, logics $\mathrm{FO}(\mathrm{Q})$ can be defined in the same way. In the following sections, we shall disallow the orcurrence of free variables in generalized quantifiers and $\mathrm{I}^{*} \mathrm{O}^{*}(\mathrm{Q})$ will denote the sublanguage of $\mathrm{FO}(\mathrm{Q})$ obtained by replacing the above formation mile for $Q_{\Lambda}$ with the following one.

- If $\varphi_{1}, \ldots, \varphi_{k}$ are formulas and, for each $i \in$ $\{1, \ldots, k\}, \bar{x}_{i}$ is a tuple of variables of arity $r_{i}$ and $f_{i}$ has exachly $r_{i}$ free variables, then $Q_{k} \bar{x}_{1} \ldots \bar{x}_{k}\left(\varphi_{1}\left(\bar{x}_{1}\right), \ldots, \bar{r}_{k}\left(\bar{x}_{k}\right)\right)$ is a formula. The latter closed formula will be refered to as a generalized atomir senteruce.

\section{Strongly Monotonic Quantifiers}

In this section. We int roduce the untion of a strongly monotonic quantifier and prove llat extensions of first-order logir: will any family of stmongly monotonic (juantifiers have a $0 / 1$ law.

Drefinition 3.1 A quantifier $Q_{\mathcal{N}}$ is sard to be strongly monotonic if for all $\tau$-s/melures $\mathcal{A}=\left\langle A, R_{1}, \ldots, R_{k}\right\rangle$

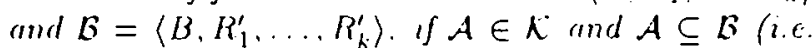
$A \subseteq B$ and $R_{i} \subseteq R_{i}^{\prime}$. for each $\left.i \in\{1 \ldots . . k\}\right)$. then $B \in \mathcal{N}$.

'Jhere are plenty of natural examples of strongly monotonic quantifiers. The most obvious strongly monotonic quantifiers are the first-order cxistential quantifier $\exists$, and the counting quantifiers $\exists i x$. meaning "there are at least $i$ distinct $x$ 's" [Imm86]. Consider now the class $\hat{\Lambda}_{\neg P}$ of non planar graphs. The quantifier $Q_{\mathcal{K}_{-F}}$ is obviously strongly monotonic: it is known ([BerT6], p. 21) that a graph is planar if it does not contain a subgraph---in the graph-theoretic sense-homeomorphic either to $K_{3.3}$ (the bi-partite 3.3 -graph) or to $K_{i}$ (the 5-clique). The notion of $n$ colorability provides us with another example. If $\mathcal{K} \neg n \mathcal{C}$ is the class of graphs that are not $"$-colorable. then $Q_{\mathcal{K}_{\neg n C}}$ is a strongly monotonic quantifier. More generally, if a property of graphs has a forbidden subgraph characterization. its complement can be expressed by a strongly monotonic quantifier. It is now folklore in the theory of random graphs that such properties are satisficd by almost no graphs [BII7?]: it is an easy consequence of the extension axioms that. for any graph $H$, almost every grapli has an induced subgrapli isomorphic to $H$. Theorem 3.1 below thus includes as subrases the results about the asymptotic probability of planarity, n-colorability, chordality, etc.

Let $Q_{s m}$ be any set of strongly monotonic quantifiers.

Theorem $3.1 F O^{*}\left(Q_{\text {.m. }}\right.$ ) has a (labllled) O/1 lau

Proof : Obriously, the sct of a.s. true or a.s false properties is closed under finite conjunctions and negations. So. since there is no free variable within the scopes of gemeralized quanrifices it is sufficient to prove that every gemeralized atomic sentence $\Phi: Q_{\wedge} \bar{x}_{1} \ldots . \bar{x}_{k}\left(\hat{r}_{1}\left(\bar{x}_{1}\right) \ldots . \bar{r}_{k}\left(\bar{x}_{k}\right)\right)$. with a strongly monotonic quantifier $Q_{\Lambda}$. has asymptotic probability 0 or 1 . If $\hat{\Lambda}=\emptyset$. then $\Phi$ is always false. and its probability is mifomly 0 . Otherwise. let $n$ be such that the probability that a $t$-structure over $n$ does not belong io $\alpha$ is $p(0 \leq p<1)$. Then, by a simple combinatorial argmment and by the strong 
monotonicity the probahility that a $r$-stmuture over $m$ (with $m \geq 2 n$ ) does not hilong to $\alpha$, is at most $\mu^{2}$. Therefore, the ratio of $\tau$-structures of Struc $n[\tau]$ which do not belong to $\alpha$ tends 100 as $n$ tends to $x$, and the asymptotic probability of $\Phi$ is 1 .

The abowe proof actually gives a more accurate insight into the asymptotic probability than Theorem 3.1 .

- The sperd of convergence of alir asimptotic probability of a sentence in $\mathrm{F}^{-}\left(\mathrm{Q}_{\mathrm{sm}}\right)$ is exponential.

- If $\boldsymbol{\Lambda}$ defines a non empry subelass, then the sentence $\left.\Phi: Q_{\kappa} \bar{x}_{1} \ldots \bar{x}_{k}(\hat{r})\left(\bar{x}_{1}\right) \ldots . s_{k}\left(\bar{x}_{k}\right)\right)$ has asymptotic probability 1.

It follows that the asymptotic probability of planarity and n-colorability of a graph is 0 . Strong monotonicity thus gives a broader language for tackling with the problem of the asymptofir probability of important properties of relational structures.

\section{Härtig Quantifiers}

This section foruses on the asymptotic probabilities of sentences with Härtig quantifiers [Här65]. IJärtig quantifiers state that two defined relations have the same cardinality. The Härtig quantifier $I_{r, w}$ is of type $\langle r . s\rangle$. i.e. it binds two formulas and $r$ variables in the first one and $s$ variables in the second one. The syntax of $\mathrm{FO}^{-}\left(I_{r . s}\right)$ is given by the new formation rule:

- if $y(\bar{x})$ and $\psi:(\bar{y})$ are 1 wo FO formulas such that the set of free variables of $q$ (resp. $\left.t^{*}\right)$ is $\bar{x}$. of length $r$ (rosp. $\bar{\eta}$. of length $s$ ), then $I_{r, s} \bar{x} \cdot \bar{y}\left(\zeta_{r}(\bar{x}), \ell \cdot(\bar{y})\right)$ is a sentence of $\mathrm{FO}^{*}\left(I_{r, s}\right)$.

Its semantios is given by:

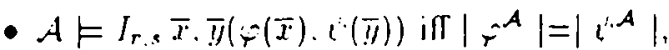
where $\bar{r}^{A}=\left\{\bar{a} \in \mathcal{A}^{r} \mid \mathcal{A} \models r(\bar{a})\right\}$ and $\ell^{A} \cdot A=\left\{\bar{a} \in A^{*} \mid \mathcal{A} \models \iota^{\prime}(\bar{a})\right\}$.

It is shown in [GT92] that. for any fixed relational schema, there is a sentenre of $\mathrm{FO}^{*}\left(I_{r . s}\right)$ that is not expressible in terms of Härtig quantifiers of type $\left\langle r^{\prime}, s^{\prime}\right\rangle$, with $\max \left\{r^{\prime} . s^{\prime}\right\}<\max \{r . s\}$. I.rt I stand for the set of Härtig (fuantifiers of any $1 y$ pr.

We next ristablish our main therem on the asymptotic probabilities of sentrures in FO*(I)

Theorem 4.1 The asymptorle probability of exry

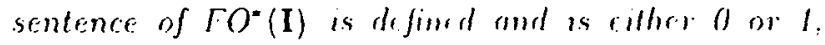
i.e. $F O^{*}($ I) has a labrlled OI/I Iari:
Proof: Without Joss of generality, we may erstrict. to generalized atomire sentences with quantifiers of the form $I_{r . r}$. Inderd. assime that $\bar{x}=\left\langle x_{1}, \ldots, x_{r}\right\rangle$ and $\bar{y}=\left\langle y_{1}, \ldots y_{s}\right\rangle$ wit./1 $r<s$. The formula:

$I\left\langle x_{1}, \ldots, x_{r}\right\rangle,\left\langle y_{1}, \ldots, y_{s}\right\rangle\left(\varphi\left(x_{1}, \ldots, x_{r}\right), \psi\left(y_{1}, \ldots, y_{s}\right)\right)$

is equivalent to:

$$
\begin{aligned}
& \quad\left\langle x_{1} \ldots x_{r}, x_{r+1} \ldots x_{s}\right\rangle \cdot\left\langle y_{1}, \ldots y_{s}\right\rangle \\
& \quad\left(\left(c_{r}\left(x_{1}, \ldots x_{r}\right) \wedge \bigwedge_{i=1}^{s-r} r_{r+i}=r_{i}\right) \cdot \iota^{\prime}\left(y_{1}, \ldots y_{r}\right)\right) .
\end{aligned}
$$

Now. Proposition 2.1 cnables us 10 reformulate our problem in combinatorial terms. Indred, for some finite sets $I$ and $J$ we have:

$$
\lim _{n \rightarrow \infty} \forall x\left(y(x) \Leftrightarrow \bigvee_{i \in I} \Phi_{C},(x)\right)=1
$$

and

$$
\lim _{n \rightarrow \infty} \forall \bar{\eta}\left(\iota^{\prime}(\bar{y}) \Leftrightarrow \bigvee_{j \in J} \Phi_{C},(\bar{\eta})\right)=1 .
$$

Therefore, the asymptotic behavior of

$$
I \bar{x} \cdot \bar{y}(\varphi(\bar{x}), \imath \cdot(\bar{y}))
$$

is identical to the asymptotic behavior of

$$
I \bar{x}, \bar{y}\left(\bigvee_{i \in J^{\prime}} \Phi_{C_{1}}(\bar{x}), \bigvee_{j \in J^{\prime}} \Phi_{C},(\bar{y})\right)
$$

with $I^{\prime}=I-J$ and $J^{\prime}=J-I$. Wir sct $\ell=N_{r}(\tau)$, $\ell_{1}=\operatorname{card}\left(I^{\prime}\right)$ and $\ell_{2}=\operatorname{card}\left(J^{\prime}\right)$. The equivalent combinatorial problem is the following:

One randomly distributes $n$ balls into $C$ equiprobable irns. For each pair $\left\langle f_{1}, \ell_{2}\right\rangle$ of integers such that $C_{1}+C_{2} \leq C_{\text {. Int }} A_{n}$ denote the probability that the number of balls in the first $\ell_{1}$ urms be equal to the number of balls in the next $f_{2}$ ones. An rasy computation vields:

$A_{n}=\frac{1}{\ell^{n}} \sum_{\substack{p=0 \\ \nu=0=n}}^{n}\left(\begin{array}{l}n \\ p\end{array}\right)\left(\begin{array}{c}p \\ p / 2\end{array}\right)\left(\ell_{1} \ell_{2}\right)^{r / 2}\left(f-\ell_{1}-\ell_{2}\right)^{n-p}$.

It is immediate that if the limit of 1, as $n \rightarrow \infty$. exists and is equal to either 0 or 1 , then FO* $(I)$ has a habelled 0/1 law. Inmma 4.2 thereafter shows that this is inderel the rase and gives also acentrate information about. the speed of enuvergence.

Lomma $4.2 \quad A, n \rightarrow \infty . A_{n} \rightarrow 0$ at erponential sperd, except when $l_{1}=l_{2}$. in which rase the speed of convergerne is $O\left(\frac{1}{\sqrt{11}}\right)$. 
Proof: See the Appendix (sertion 1.1).

As an aside. the next proposition shows that the complexity of the decision problem for the asymptotic truth of a sentence of $\mathrm{FO}^{*}(\mathbf{I})$ is a plexity of the counterpart problem for FO [(iras:3].

Proposition 4.3 The deristun problem for the inalue of the asymptotic probululilits of sententits of $F O^{*}(\mathbf{I})$ is PSPACE-complete.

Proof : It relies on the faret that the value. of the asymptotic prohilility of a sontence of $\mathrm{FO}^{*}(\mathbf{I})$ depends only on the valurs of asymptotic probabilities of FO sentences. Imlend. a sminmes of the form $I_{r, r} \bar{x} \cdot \bar{y}(r(\bar{x}) \cdot 2 \cdot(\bar{y}))$ has asymptot in probability 0 (1) iff $\lim _{n \rightarrow \infty} \forall \bar{x}(\because(\bar{x}) \Leftrightarrow(\cdot \bar{x}))=0(1)$.

If free variables can ocour within the scope of a Härtig quantifier, the $0 / 1$ law (and cren the limit law) fails.

Theorem 4.4 There is a sentence $\hat{y}$ of $F O(\mathrm{I})$ whost probability $\mu_{n}(\varphi)$ does not hare a limit.

Proof: It is sufficient to show that the property of graphs, expressible in $F O(I)$. that says "there is at least) one vertex adjacent to exactly the half of the other vertices" does not have an asymptotic probability. Indered. while the sentener is obvionsly always false on graphs of even oreder. its probability does not tend to 0 , as $n \rightarrow \infty$, on graphs of order $2 n+1$. To see this last point. we shall rely nn a general theorm given in [Bol85)] (Chap. III. 'Thmorm 1. p. ji). which asserts in particular the following

Proposition 4.5 Let $\mathcal{G}(n, p)$ lir the model ronsist-

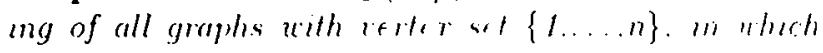
the edges are chosen indryendinlly wilh problablatyp. which can be a function of $n$. l.et e $>0$ bre firted and $\epsilon n^{\frac{-3}{2}} \leq p \leq 1-\epsilon n^{\frac{-3}{2}}$. Let $k=h(n)$ be a notural number and set $\lambda_{k}(n)=n b(k: n-1.1)$. where

$$
b(j: n \cdot p)=\left(\begin{array}{l}
n \\
j
\end{array}\right) r^{\prime}(1-p)^{n-\prime} .
$$

Denote also by $\mathrm{X}_{k}$ the ramdom errable replesenting the number of restices of drame $h$ in a random graph. One sces that $\lambda_{k}(n)$ is errertlil the erpertation of $\mathrm{H}_{k}$. Then the following property holds.

$$
\text { If } \lim _{n \rightarrow \infty} \lambda_{k}(n)=\infty \text {, then } \lim _{n \rightarrow-\infty} \operatorname{Proh}\left(x_{k} \geq 1\right)=1 \text {. }
$$

for every fircd $\mathrm{C}$. lluss. we are in a position to apply l'roposition 1.5. with $p=\frac{1}{2}$. for graphs of order $2 n+1$. I sing Stirling $s$ formula. We have immerliately

$$
\lambda_{n}(2 n+1)=(2 n+1)\left(\begin{array}{c}
2 n \\
n
\end{array}\right) \cdot 1^{-n} \sim 2 \sqrt{\frac{n}{\pi}} \rightarrow \cdots
$$

The probability $\mu_{n}(\xi)$ of the senteme $\hat{y}$ of $\mathrm{FO}(\mathbf{I})$, which we have comsidered, has no proper limit, but takes. as $n \rightarrow x$. (only) the two values 0 and 1 . 'This concludes the pronf of Theorem 4.4

The same technique can be applind to similarity quantifiers $S_{r}$, of ype $\langle r, r\rangle$, which state that two defined relations are isomorphic, and not only of equal cardinalities (Härtig quantifiers). The syntax is similar to Härtig quamtifiers with $S$ in place of $I$. The smmantios is defined as follows:

$$
\text { - } \mathcal{A} \vDash S_{r} \bar{x}, \bar{y}\left(\zeta^{\prime}(\bar{x}) \cdot \iota^{\prime}(\bar{y})\right) \text { iff }\left\langle A, Y^{\mathcal{A}}\right\rangle \cong\left\langle\mathcal{A}, \iota^{\mathcal{A}}\right\rangle \text {. }
$$

Let $\mathbf{S}$ be the set of similarity quantifiers of all types. We define $F O^{*}(\mathrm{~S})$ in the same way as $F O^{*}(\mathbf{I})$.

Corollary 4.6 The asymptotic mobrability of every sentence of $F O^{*}(\mathrm{~S})$ is defined and is either 0 or 1 , i.f. $F O^{*}(\mathrm{~S})$ has a lalu llad O/1 law.

The proof follows from Theorem 4.1. Indeed, if $\lim _{n \rightarrow \infty} \forall \bar{x}(\infty(\bar{x}) \Leftrightarrow i(\bar{x}))=0$. then the probability liat $\left|F^{A}\right|=\left|i^{A}\right|$ (which majorates the probabiling that $\left.\left\langle A . \mathcal{S}^{A}\right\rangle \cong\left\langle A, \psi^{A}\right\rangle\right)$ goes to 0 when $n \rightarrow \infty$. Otherwise. if $\lim _{n \rightarrow \infty} \forall \bar{x}\left(\varphi(\bar{x}) \Leftrightarrow t^{\prime}(\bar{x})\right)=1$, then $\lim _{n \rightarrow \infty} S_{r} \bar{x}, \bar{y}(\varphi(\bar{x}) \cdot \iota(\bar{y}))=1$.

\section{Rescher Quantifiers}

We now proced to the study of a natural extension of $\mathrm{FO}^{*}(\mathbf{I})$ including the possibility of testing incqualities of cardinalities of first-order definable relations. The corresponding cluantifiers is refered to in the lit-

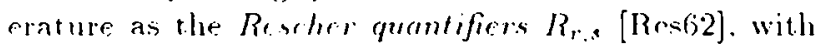
$R_{r . .}$ of type $\langle r, s\rangle$. Inre again, the syntax of $\mathrm{FO}^{*}(R,, s)$ is given by the new formation rule:

- if $\varphi(\bar{x})$ and $\varphi(\bar{y})$ are two $\mathrm{F}()$ formulas such that the set of free variables of $\varphi$ (resp. $\psi$ ) is $\bar{x}$. of lengll, $r$ (ms) $\bar{y}$. of longth s). then

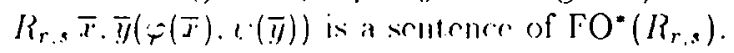

Its segmalltirs is given by:

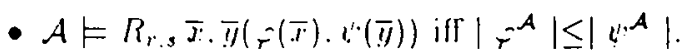


Obviously, llee 0/1 law firils in this new romtext. If $P$ is a inary predicate. the as meptotir probiabil-

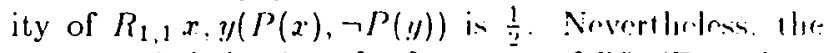
asymptotic behavior of a fragment of $F()^{*}(R)$. where R. stands for the set of Rreshere quantificrs of all types, is fairly regular, as shmon by the next lluersrem. Rerall that a senteure of $\mathbf{I}^{*}(\mathbf{R})$ of the from $R_{r, s} \bar{x}, \bar{y}\left(s(\bar{r}), e^{\prime}(\bar{y})\right)$ is callorl y ge nerolized atomic senttence.

Theorem 5.1 The asymplotir probralilily of rery generalized atomic sentenre of $F^{*}(\mathbf{R})$ is defined and its value ranges over $\left\{0, \frac{1}{2}, 1\right\}$.

Proof: It goes along the same limes as the proof of Theorem 4.1. We consider generalized atomic srintences like $R_{r, r} \bar{x}, \bar{y}(\varphi(\bar{x}), v(\bar{y}))$. Once again. we reformulate our problem:

One ranclomly distributes $n$ balls into $t$ equiprobable urns. For earh pair $\left\langle\ell_{1}, \ell_{2}\right\rangle$ of integers such that $\ell_{1}+f_{2} \leq f$. let $B_{n}$ denote the probability that the nmmber of balls in the first $\ell_{1}$ urns be lesser than the number of balls in the next $\mathcal{L}_{2}$ ones. In easy computation yiclds:

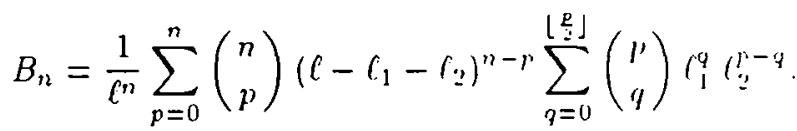

Clearly, if $\lim _{n \rightarrow \infty} B_{n}$ always exists and bolongs to a set $\mathbf{B}$, then the asymptotic probability of all sentences of the form $R_{r . r} \bar{x} \cdot \bar{y}\left(r^{-(\bar{x})} \cdot e^{\prime}(\bar{y})\right)$ exists and belongs to $\mathbf{B}$. The next lemma explicitly describes $\mathbf{B}$. hence completing the proof of Theorem j.l

Lemma 5.2 As $n \rightarrow x \cdot B_{n} \rightarrow 0$. $\frac{1}{2}$ or 1 .

Proof : See the Appendix (snetion 1.2)

The limit law of Theorem 5.1 (an be rasily generalized to sentences of $\Gamma O^{-}(R)$ with ane gencralized atomic sub-sentence. or under some tedmical assumptions to sentences with several grneralized atomic subsenteness, with dyadie limit law. We emjerenture that in the general case. $\mathrm{FO}^{-}(\mathbf{R})$ almits a dyalie limit law Note that if the existence of the asymptotie molnahility for all sentemes of $\mathrm{FO}^{*}(\boldsymbol{R})[\bar{T}]$ is estahlishel the

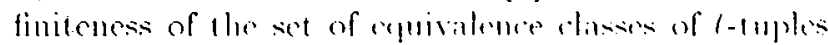

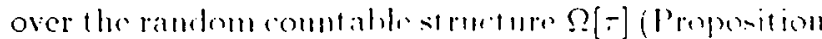

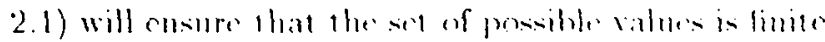
for carle $\tau$.

\section{Conclusions}

Wie undertook a study of the asymptotic truth of sminnres for extensions of FO with generalized quantifiers. and came up with a characterization of the ay ymptotic behavior of two kinds of languages. corresponding on the one hand to strongly monotonic guantifiers and on the other hand to counting quantifiers. As for $\mathrm{FO}^{*}(\mathrm{I})$. the syntactic constraint (no free variables within the srope of Härtig quantifiers) we imposed on the formulas revealed essential: our counterexample to the $0 / 1$ law for the full language $\mathrm{FO}(\mathbf{I})$ iudicates the very place where the boundary of $0 / 1$ laws is broken in the hierarchy of counting extensions of $1: 0$

In most cases. He 0/1 law no longer holds for FO(Q) where $Q$ is a (set of) generalized quantifier(s). This is tlie case for instance of partially ordered cuanlifiers. surh as Henkin quantufrers [Hentil]. which yich the same expressive power as existemtial second-order logir [BCisic]. For example, the following sentence expresses the fact that the cardinality of the domain is even

$$
\left(\begin{array}{c}
\forall x \exists r \\
\forall y \exists u
\end{array}\right)\left(\begin{array}{c}
(x=y \Leftrightarrow u=u) \wedge \\
(x=u \Leftrightarrow y=v) \wedge \\
(v \neq x))
\end{array}\right)
$$

Indeed, it states that there exist two mary functions $f$ and g. which are equal and one-one: since the domain is finte. lhey define a bijection. Which is involutive and has no fixpoint.

In the body of the paper. we did not consider langunges of the form $\mathcal{L}^{-}(Q) \cdot \mathcal{L}$ being a logic stronger than FO. or non uniform probability measures. It is rear that the asymptotic probabilities of sentences are similar if $F()$ is replaced with FP. namely firstorder logi: plus a positive fixed-point operator. There are wo basie reasoms for that: (i) each FP formula with / free variahles is asymptotically equivalent to a union of equivaleme vasses of (-iuples of the random countahlestructure $\Omega$, thus ensuring that Proposition 2.1 applies to Fl: (ii) all variables of an expression $Q, \ldots, \bar{x}_{1} \ldots \bar{x}_{1}\left(\hat{-1} \ldots ., \hat{r}_{p}\right)$ occurring in a formula of $F \cdot P\left(Q_{2}, \ldots\right)$ or $F=(I)$ are bound. so the fixed-point combrume rammot "imnolve" a gemeralized quantifier As for $I_{\text {in }}\left(Q_{\text {s.m }}\right)$. olviously, it does not have a $0 / 1$ law. the parity of the domain is expressible in $\operatorname{Is}_{\sim_{u}}^{u}(C)$. where $C$ stands for llw set all counting quantifiers $\exists i x$

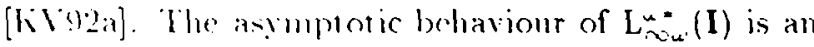
open yulesion. 


\section{Acknowledgements}

The authors wish to thank Yuri Ciurevieh for fruitful discussions.

\section{A Appendix}

\section{A.1 Proof of Lemma 4.2}

We suppose that $n$ is even. 'This restriction is harmless and the case $n$ odd can he cloalt with in an analogous way.

Define $\alpha=\ell_{1} / \ell, \beta=\ell_{2} / \ell, n=2 k$ and $p=2 u . A_{n}$ can thus be rewritten as

$A_{n} \equiv \tilde{A}_{k}=\sum_{u=0}^{k}\left(\begin{array}{l}2 k \\ 2 u\end{array}\right)\left(\begin{array}{c}2 u \\ u\end{array}\right)(n \cdot 3)^{u}\left[(1-\alpha-\beta)^{2}\right]^{k-u}$ i.e.

$$
\tilde{A}_{k}=(2 k) ! \sum_{u=0}^{k} \frac{(\alpha / \beta)^{u}\left[(1-\alpha-\beta)^{2}\right]^{k-u}}{(u !)^{2}[2(k-u)] !}
$$

Setting also

$$
\begin{aligned}
& \tilde{A}_{k} \stackrel{\text { def }}{=}(2 k) ! B_{k} \\
& B(z) \stackrel{\text { def }}{=} \sum_{j=0}^{\infty} B_{j} z^{2 j} . \text { so that } . \tilde{i}_{k}=\frac{d^{2 k} B(z)}{d z 2 k} \mid==0
\end{aligned}
$$

and using the convolution character of $\tilde{f}_{k}$, one notices that $B(z)$ can be expressed in terms of the product of the two following series:

1. the classicaly so-callod modified Bessel function

$$
\mathbf{J}_{0}(i, r) \equiv \mathbf{I}_{0}(x)=\sum_{j=0}^{\infty} \frac{1}{(j !)^{2}}\left(\frac{x}{2}\right)^{2 . j}
$$

2. the serics

$$
F(z) \stackrel{\text { def }}{=} \sum_{j=0}^{\infty} \frac{x^{2 j}}{(2 j) !}=\frac{1}{2}\left[c^{x}+e^{-5}\right] .
$$

'This yickls clirectly'

$$
B(z)=\mathbf{I}_{0}(2 \sqrt{a .3}:) F[(1-a-\beta) z] .
$$

We now nse Sonine's integral representation [LSTit]

$$
\mathbf{I}_{0}(z)=\frac{1}{2 i \pi} \int_{\left|\omega_{\mid}\right|=1} \frac{e^{\frac{12}{ \pm}\left(\omega^{\prime}-\frac{1}{\omega}\right)}}{\omega} d \omega .
$$

'Tlien, by a symmetry argument with respect to 2 , it follows that

$$
B(z)=\frac{1}{2 i \pi} \int_{|\omega|=1} \frac{e^{i\left[i \sqrt{\alpha \beta}\left(\omega-\frac{1}{\omega}\right)+1-\alpha-\beta\right]}}{\omega} d \omega
$$

Finally, making ther (hange of variable $2=e^{i \theta}$, we get

$$
\tilde{A}_{k}=\frac{1}{\pi} \int_{0}^{\pi}[2 \sqrt{\alpha 3} \cos \theta+(1-\alpha-\beta)]^{2 k} d \theta \text {. }
$$

It is known that $\tilde{A}_{k}$ can be expressed directly in terms of the Legendre fumctions of first and second kind. according to the values of the parameters. It is nevertheless here more direct to apply the saddle point method, see e.g. [LSTi], which produces an asymptotic expansion for integrals of the form

$$
I I(\lambda)=\int_{a}^{b} e^{\lambda f(x)} d x, \text { as } \lambda \rightarrow x .
$$

We expedite first two borderline cases

i) $\alpha \beta=0$, say for instance $a=0$. Then

$$
\bar{A}_{k}=(1-3)^{2 k} \text {. }
$$

which tends to zero exponentially fast.

ii) $\alpha=\beta=1 / 2$. Then

$$
\tilde{A}_{k}=\frac{(2 k) !}{4^{k}(k !)^{2}} \sim \frac{1}{\sqrt{k \pi}}\left[1+O\left(k^{-1}\right)\right],
$$

by using Stirling s formula.

Assuming $\alpha \beta \neq 0$ and $\alpha, 3$ not both equal to $1 / 2$. we have

$$
\tilde{A}_{k}=\frac{1}{\pi} \int_{0}^{\pi} t^{k}\left[\log [2 \sqrt{a 3} \cos \theta+(1-\alpha-3)]^{2}\right] d \theta .
$$

which is of the form 1 . The behavior of $\dot{A}_{k}$. as $k \rightarrow x$ clepends on the maximum of the function

$$
|2 \sqrt{n \cdot 3} \cos \theta+(1-a-3)|
$$

and on the position of the point, say $\theta_{0}, 0 \leq \theta_{0} \leq \pi$. where this maximum is attained. Here $\theta_{0}=0$ is an end of the interval of integration. Then we get approximately one half of a Gaussian integral, see [LSTi], so that

ik.

$\frac{\left[1-(\sqrt{0}-\sqrt{3})^{2}\right]^{2 k}}{2} \sqrt{\frac{1-(\sqrt{0}-\sqrt{3})^{2}}{2 k \pi \sqrt{a 3}}}\left[1+O\left(\frac{1}{k}\right)\right]$.

showing again the convergence to 0 at exponential speed. The proof of the lemma is concluded. 


\section{A.2 Proof of Lemma $\mathbf{5 . 2}$}

We use the same notation as in the proof of lemma 4.2. It follows that

$$
B_{n}=\sum_{r=0}^{n}\left(\begin{array}{l}
n \\
p
\end{array}\right)(1-a-.3)^{n-r}(n+\beta)^{r} \int_{r} \text {. }
$$

where

$$
f_{p}=\sum_{q=0}^{\left\lfloor\frac{p}{q}\right\rfloor}\left(\begin{array}{l}
p \\
q
\end{array}\right)\left(\frac{n}{a+3}\right)^{4}\left(\frac{\beta}{n+\beta}\right)^{p-q} .
$$

From Toeplitz Irmma, ser [Ineti], it follows that if $f_{p}$ tends to a limit as $p \rightarrow x$, then $B_{p}$, will tend to the same limit. It turns out that $f_{p}$ can be expressed in an integral form. allowing to use the saddle-point method. see [LSTi]. Introduce the cumulative distribution function of the binnmial distribution

$$
B(k: p, r)=\sum_{p=0}^{k}\left(\begin{array}{l}
p \\
q
\end{array}\right), s^{p-q}
$$

where $0 \leq k \leq p, 0 \leq r . s$ and $r+s=1$. Then $f_{p}=B\left(\left\lfloor\frac{\bar{p}}{2}\right\rfloor: p, r\right)$. It is sweet to remark that $B(k ; p, r)$ admits the following integral representation, see $[\mathrm{Fel} T 0]$.

$$
B(k: p, r)=(p-k)\left(\begin{array}{l}
n \\
p
\end{array}\right) \int_{0}^{*} t^{p-k-1}(1-t)^{k} d t
$$

Assume $p$ is odd. for instance $p=2 u+1$, and set $s=\frac{3}{a+3}$. Then

$$
f_{p} \stackrel{\operatorname{dof}}{=} f_{u}=\frac{(2 u+1) !}{(u !)^{2}} \int_{0}^{\frac{3}{u+4}} t^{u}(1-t)^{u} d t \text {. }
$$

As in the proof of lemma 4.2. we are in a position to apply the saddle point mothod to the above integral, which yields three different rases. according to the position of the point $t=1 / 2$ (which is the point where the function $\operatorname{lng} f(1-t)$ reacliss its maximum) with respect to $s$ :

1. $\beta>\alpha$. Denoting by $I(11)$ the integral coming in $\tilde{f}_{u}$ we obtain

$$
I(u) \sim \frac{4^{-u}}{2} \sqrt{\frac{\pi}{u}}\left[1+O\left(u^{-1}\right)\right] .
$$

so that. aftor applying stirling s formula to cyalwate the confficient $\frac{f_{u t}}{f(u)}$.

$$
\tilde{f}_{u} \sim 1+0\left(\frac{1}{u}\right)
$$

2. $\beta<a$. Here, on $[0, s]$, the function $\log t(1-t)$ has its maximum at $t=s$, so that $\dot{f}_{u} \sim$

$\left[\frac{4 \alpha \beta}{(\alpha+\beta)^{2}}\right]^{u} \frac{2^{3 / 2} \alpha \beta}{\sqrt{\pi u\left(\alpha^{2}+\beta^{2}\right)(\alpha+\beta)}}\left[1+O\left(\frac{1}{u}\right)\right]$, which tends to 0 exponentially fist.

3. $\beta=\alpha=1 / 2$. Now we obtain a quantity rquivalent to the arca of a scmi-gaussian and

$$
\tilde{f}_{u} \sim \frac{1}{2}+O\left(\frac{1}{u}\right)
$$

By mimicking the above derivation. one can see that the same limits hold in the case $p=2 u$. Irnce $f_{p}$, and consequently $B_{1}$. have, when $p \rightarrow \infty$. one of the limits 0,1 , or $\frac{1}{2}$. drpending on the respertive values of $n$ and 3 . The Irmma is proved.

\section{References}

[AV'91] S. Abitchoul and V. Vianu. Generic computation and its complexity. In Proc. 23rd ACM Symp. on Throry of Computing (1991) 209-219.

$[$ Ber 76$]$

C. Berge. Graphs and hypergraphs. North-IIolland mathematical library, Vol. 6 (North-Holland, Amsterdam, 2nd edition. 1976$)$.

[BF85] J. Barwise and S. Feferman, editors. Model theoretic logics. Perspectives in Mathematical Logic (Springer Vorlag. Berlin, 1985).

[BG86] A. Blass and Y. Gurevich. Henkin quantifiers and complete problems. Annals of Pure and Applical Logir 32 (1986) $1 \cdots 16$.

[IBCik85] A. Blass. Y. Curevich, and D. Kozen. A zero-one law for logic with a fixed-point operator. Information and ('ontrol 67 (1985) $70-90$

[I3HTSS] A. Blass and F. IIarary. Properties of almost all graphs and complexes. Inumal of Groph Theory $3(1979) \cdot 225 \cdot 240$.

[Bol85] B. Bollobas. Random Cirophs (Academic: Presis. 1985)

[Fagio] R. Fagin. Probabilities on finite models. Joumul of siymbolis logu $41(1)(1970) 50$ 58. 
[Fag90] R. Fagin. Finite moled thoory--a personal perspective. In S. Abitoboul and P. Kandlakis. editors: Proc. 3nd lat. Conf. on Database Thene. Inture Vores in Computer Science. Vol. 170 (Springer-Vorlag. Berlin, 1990) 321.

[Fel70] Wr. Feller. An intirnturtoon to Probatility Theory and ats Ipplicatoms, volume 1. (Wiley, 3rd edition. 1950).

[GKLT69] Y'. CIlcbskii. D. Kogan. M. I.iogon kil. and V. Talanov. Range and degree of realizability of formulas in the restricted predicate calculus. Kilrermetica 2 (Kiver. 1969) $17 \cdot 28$. Fnglish transiation. Cubremetirs 5 (1969) $1.12-151$.

[Gra83] E. Crandjean. Complexity of the first order theory of almost all strurtures. Information and contwol 52 (198:?) $150-20.4$.

[GS86] Y. Gurevich and S. Silnlalt. Fixnd-point. extensions of first oriter logic. Ammals of Pure and Aplied I.ngur 32 (1986) 265-280.

[GT92] S. Grumbach and C. Tollu. Query languages with commors. In J. Biskup and R. IIull. editors: Pror. ${ }_{1}$ th Int. Conf. on Dalabase Thromy. I.roture Notes in Computer Science. Vol. 616 (Springer-Vingg. Berlin. 1992) 12.1-1:39.

[Här65] H. Härtig. Ïher rimen Quamifikator mit zwei Wirkungsbmprichrn. In L. Kalmar, editor: Collogumm nn Fommdations of Wathematirs. Wathe matical Marhanes and llew

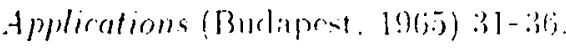

[Hel92] I. Mrlla. Iogional hirmahies in PTWV:. In

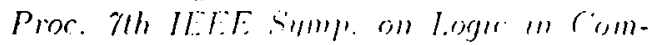

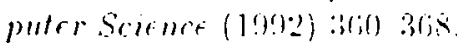

[Hen61] I. Henkin. Someremartion infinim long formulas. In Infmelalie methorls. Proce Symp. Foundations of Molliematien. Marsau (Pergamnn Pros. I.ondon. 1!16i1) 167\% 183.

[Imm86] ¿. Immerman. Rrlalimal curries computable in polymomial time. Information and Control $68(1 !) \times(j) \times(j \cdot 10)$.

[Kiny90] V. Linyazev. Znen-one law for an extension of first-order predirate langnage. Kybernetika 2 (Kiev. 19!)()) 1]()-113. Fughish

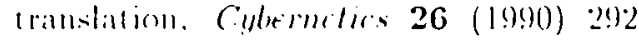
2 () 6 .

[KVit] P. Rolation and M. V. Vardi. The derision problem for the probabilities of higher-


on Theorly of Computing (1985) 125-435.

[KI90a] P. Folatitis and M.Y. Vardi. 0/1 laws and decision problems for fragments of secondorder logic. Information and Computation $87(1090) .302-3.38$.

[KV90b] P. Kolaitis and M.Y. Vardi. 0/1 laws for infinitary logic. In Proc. sth IEEE Symm). on Logir in Computer Srirnee (1990) 150 167 .

[KV92a] P. Kolaitis and J. Vänänen. Cieneralized quantifiers and pebble games on finite structures. In Proc. 7th IEEE Symp. on Logic in Computer Srienre (1992) 348-359.

[KV:2b] P. Kolait is and M.Y. Vardi. Fixpoint logic v's. infunitary logic in finite-model theory. In Proc. 7th IEEE Symp. on Logic in Computer Srience (1992) 46.57.

[Loeti] $\quad 11$ Loeve. Probability Theory I. (SpringerVerlag. Berlin, 1977).

[LSTT] M. Lavrentiev and B. Shabat. Méthode de la Theorie diune Fonction d'une Variable Complere. (MIR, Moscon. 197T).

[Lin66] P. Lindström. First order predicate logic with generalized quantifiers. Theoria 32 (1966) $186-195$.

[Mosij] A. Mostowski. On a generalization of quantifiers. Fundamenta Mathematica 44 (195T) $12 \cdots 36$.

[Rrifi2] N. Rescher. Plurality quantification. Journal of Siymbolir. Logir 27 (1062) 373-374. 
Unité de Recherche INRIA Rocquencourt

Domaine de Voluceau - Rocquencourt - B.P. 105 - 78153 LE CHESNAY Cedex (France)

Unité de Recherche INRIA Lorraine Technopôle de Nancy-Brabois - Campus Scientifique 615. rue du Jardin Botanique - B.P. 101 - 54602 VILI.FRS LES NANCY Cedex (France)

Unité de Recherche INRIA Rennes IRISA. Campus Lniversitaire de Beaulieu 35042 RENNE.S Cedex (France) Unité de Recherche INRIA Rhône-Alpes 46, avenue Félix Viallet - 38031 GRENOBLE Cedex (France)

Unité de Recherche INRIA Sophia Antipolis 2004, route des Lucioles - B.P. 93 - 06902 SOPHIA ANTIPOLIS Cedex (France)

\section{EDITEUR}

INRIA - Domaine de Voluceau - Rocquencourt - B.P. 105 - 78153 LE CHESNAY Cedex (France)

ISSN $0249-6399$

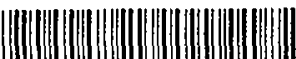

